\title{
Insight into the Structure and Nanoscale Conductivity of Fluorinated Ionomer Membranes
}

\author{
Renate Hiesgen, ${ }^{\mathrm{a}, *, \mathrm{z}}$ Tobias Morawietz, ${ }^{\mathrm{a}}$ Michael Handl, ${ }^{\mathrm{a}}$ Martina Corasaniti, ${ }^{\mathrm{b}}$ \\ and K. Andreas Friedrich ${ }^{\mathrm{c}, *}$
}

${ }^{a}$ University of Applied Sciences Esslingen, Esslingen 73728, Germany

${ }^{b}$ Solvay Specialty Polymers Italy S.p.A., Bollate (Mi) 20021, Italy

${ }^{c}$ German Aerospace Center, Institute of Technical Thermodynamics, Stuttgart 70569, Germany

\begin{abstract}
A material-sensitive atomic force microscopic (AFM) tapping mode was combined with current measurements to investigate structure, phase separation, and conductive structure of surfaces and cross sections of long side chain Nafion and short side chain AQUIVION PFSA ionomer membranes. We found unexpected large-scale ordered structures consistent with a dominant lamellar polymer structure at the cross sections. The highly terraced areas of both ionomers have a wide distribution of layer thicknesses from sub-nanometer to a few nanometers. In both broad size distributions, preferential sizes were identified that reflect the different lengths of the molecular side chains, indicating a stacking in layers. The nanoscale phase separation of the ionomer was analyzed by using the capacitive current distribution. In AQUIVION PFSA, larger connected water-rich ionic areas were found than in Nafion with same total ionic area. A steady-state current at the cross sections evolved only after an activation period by enforcing current flow though the membrane. A comprehensive and heterogeneous current distribution was observed with highly conductive areas. In contrast, on outer membrane surfaces, only non-continuous spot-like currents were observed. In general, our measurements are consistent with conduction in water layers in-between polymer chains and a bi-continuous structure under faradaic current flow. (C) The Author(s) 2014. Published by ECS. This is an open access article distributed under the terms of the Creative Commons Attribution Non-Commercial No Derivatives 4.0 License (CC BY-NC-ND, http://creativecommons.org/licenses/by-nc-nd/4.0/), which permits non-commercial reuse, distribution, and reproduction in any medium, provided the original work is not changed in any way and is properly cited. For permission for commercial reuse, please email: oa@electrochem.org. [DOI: 10.1149/2.0701412jes] All rights reserved.
\end{abstract}

Manuscript submitted May 30, 2014; revised manuscript received August 21, 2014. Published August 29, 2014. This was Paper 1338 presented at the San Francisco, California, Meeting of the Society, October 27-November 1, 2013.

Perfluorinated sulfonated ionomer membranes (PFSA) have been established as proton-conducting electrolytes in many technical applications, most notably in brine electrolysis but also with increasing importance as electrolytes for fuel cell applications. For highperformance operation of fuel cells, the water management of the membrane is of great importance. Therefore, the nature of water transport and the conductive structure of the ionomer membrane are still under investigation. In particular, the nanoscopic ionomer properties at non-equilibrium conditions under steady-state current flow close to operation conditions are expected to differ from equilibrium state and have not been characterized in detail.

During solidification of perfluorinated polymers (PFSA) casted from dispersions, a phase separation occurs that is the key factor in providing ionic conductivity. The hydrophilic sulfonate-terminated end groups of the side chains cluster together to form an ionic phase. The perfluorinated molecular backbone is assumed to form bundles due to the hydrophobic interaction and to undergo partial crystallization that is considered to enhance mechanical strength. ${ }^{1}$ The size of these crystalline areas, as determined from scattering data, is reported to be within 0.5 to $10 \mathrm{~nm}^{2,3}$ The spacing of the average hydrophobic/hydrophilic separations was obtained from the position of the ionomer peaks in scattering data and determined to approximately 3-7 nm depending on the water content. ${ }^{4}$ While the hydrophobic phase provides mechanical stability, the ionic phase provides a waterbased proton-conducting network. It is generally assumed that this ionic phase forms a continuous conducting network. Under fuel cell operating conditions this assumption must be valid, but at ex-situ equilibrium conditions without current flow this may not be the case. Most of the analytical studies have investigated ionomer membranes under the latter conditions.

Perfluorinated polymers with different lengths of side chains are in use. Nafion, with the longest side chain, is the most commonly used membrane. The shortest side chain is present in the AQUIVION PFSA membrane. The molecular structures of Nafion and AQUIVION PFSA are shown in Figure 1. The density of side chains defines the equivalent weight that is a measure for the ionic strength. The length of the side chains determines the phase separation and therefore has an influence on physical and chemical properties, i.e degree of crystallinity and mechanical strength. 2,5,6

Although Nafion is a well-known ionomer material used for many decades as polymer electrolyte, there is still debate regarding its nanostructure. The generally assumed mostly amorphous structure with a crystalline content of approximately $20 \%$ is a result of structure determination by scattering techniques. The most detailed information has been retrieved from small angle X-ray scattering (SAXS) and related techniques. In all cases, a model must be fitted to the scattering data within a broad range of humidity. Different models have been found that match the experimental data. The formation of ionic clusters in Nafion was first described by Gierke et al. ${ }^{7}$ based on the Eisenberg description of polymers. The reduction of the total free energy of the system is described by the formation of ionic clusters dispersed in a hydrophobic polymeric matrix. ${ }^{8}$ Gierke assigned a size of approximately $4-5 \mathrm{~nm}$ to these ionic clusters, as determined from the position of a broad peak in the pattern of the scattering data; the so-called "ionic peak" exhibits characteristic changes with water content. It is generally assumed that the detailed nanostructure depends strongly on temperature and humidity and is also affected by pretreatment as described, e.g., by Alberti et al. ${ }^{9}$ A prominent model in the literature proposes flat polymeric fibrils and bundles of aggregated polymer backbones with a bundle size of approximately $3-4 \mathrm{~nm} .{ }^{10-12}$ These bundles are aggregated to aligned structures of approximately $50 \mathrm{~nm}$ based on scattering data. In this model, the water surrounds the fibrillar objects and is not confined to pores. Another model based on additional arguments of electrostatic stability and the linear dependence of the ionomer peak position with water content is the formation of thin water sheets in-between layers of backbone phase. ${ }^{4}$ A lamellar structure has been proposed in 1980 by Falk et al. ${ }^{13}$ and later also by $\mathrm{Litt}^{14}$ in order to explain the swelling behavior of Nafion. In a further refinement a sandwich-like model was developed in 2001 by Haubold et al. ${ }^{15}$ Parallel cylindrical water nanochannels with a size of $2.4 \mathrm{~nm}$ for the inner water channel have been introduced by Schmidt-Rohr and Chang ${ }^{16}$ but have recently been shown by Kreuer and Portale ${ }^{4}$ to be unsuitable to describe a large range of experimental data in varying humidity. Recently, more complicated model structures have been published that propose a bi-continuous network from an ionic phase and a partially crystalline hydrophobic phase. ${ }^{17}$ 

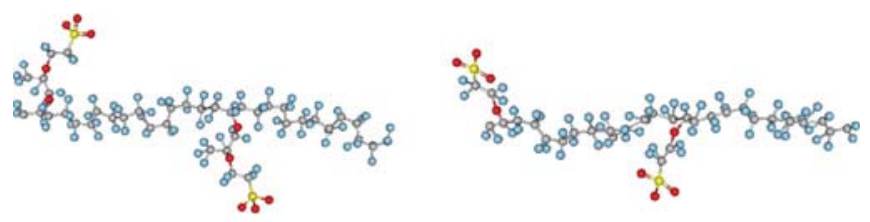

Nafion ${ }^{\circledR} 212$

AQUIVION ${ }^{\circledast}$ PFSA E87

Figure 1. Structure of two Nafion and AQUIVION PFSA polymer backbone units with side chains.

The difference in surface energy between the ionomer and the environment at solidification leads to the formation of a surface skin layer, as proposed by several authors and based on different experiments. ${ }^{18,19}$ From SAXS measurements and modeling of these data, a thickness of approximately $5 \mathrm{~nm}$ was estimated. A $4.5 \mathrm{~nm}$ thick crystalliterich surface layer with a $40 \%$ higher degree of crystallinity based on synchrotron grazing incidence X-ray diffraction (GIXRD) and small angle X-ray scattering (GISAXS) measurements with crystallites oriented parallel to the surface was reported. ${ }^{20}$ At the ionomer/water vapor interface, a hydrophobic surface is observed where the molecular backbone is present at the outer surface and the ionic side chains are switched to the interior. This interface is proposed to consist of polymer bundles predominantly oriented parallel to the surface, where, besides a few defects, no ionic channels have an exit for water/proton exchange with the environment. ${ }^{21}$ An interchange of water from the outside to the interior is possible only through defects with a low overall conductivity, as detected with material-sensitive tapping mode AFM. A skin layer thickness of $5 \mathrm{~nm}$ could be measured for Nafion NR212. ${ }^{22,23}$ Upon contact with water, the ionic side groups can change their orientation within seconds, and the surface becomes hydrophilic. ${ }^{18}$ At the interface with water, the structures tend to orient vertically to expose ionically conductive channels from the bulk to the exterior that supply an exit for water/proton exchange.

Nafion structures have been directly visualized by transmission electron microscopy (TEM), where the nano-sized domains can be directly imaged. ${ }^{24,25}$ In dry membranes from $3 \mathrm{M}$ polymers (side chain with medium size), an even distribution of ionic clusters with a size of 2 $\mathrm{nm}$ and an inter-cluster distance of $3 \mathrm{~nm}$ was found, a value also typical for the other ionomer systems. ${ }^{26}$ By partially hydrated cryo-TEM investigation, a swelling of the cluster size was observed until they started to coalesce without changing their distances. A disadvantage of TEM investigation of the PFSA ionomer is its restriction to dry or frozen state of the ionomer and it is prone to cause radiation damage to the polymer. In addition, only very thin films can be investigated because the film thickness is limited by the transmission range of the electron beam. Since a change in properties for thin films has been found, the results may not be valid for thicker films that are used, e.g., as electrolyte membranes for fuel cells.

Scanning force microscopy also enables direct imaging, and several studies of ionomer membrane surfaces have been performed. Here, the evaluation of the phase shift signal in tapping mode scanning force microscopy (AFM) leads to a visualization of cluster-like struc- tures a few nanometers in size depending on the ambient conditions and the applied force. ${ }^{27,28,29,30}$ In addition, conductive AFM provides information on nanoscale conductivity. An important advantage for fuel cell membrane characterization is the possibility to image under non-equilibrium steady-state conditions with current flow. ${ }^{31,32,22,33}$

Furthermore, with advanced AFM tapping methodology, it is possible to study local mechanical properties in correlation with topography data. Of main interest for ionomer investigation are the local adhesion force, the stiffness (based on DMT-modulus), the surface deformation, and the energy dissipation. These properties are characteristic for different materials. In an earlier study it has been shown that an identification of the phase separation into hydrophobic or hydrophilic phases on an ionomer membrane is possible. ${ }^{22}$

In this paper, we report for the first time direct imaging by AFM of lamellar nanoscale structure of AQUIVION PFSA and Nafion ionomer membranes, which have been obtained by investigating cross sections of the membranes. Furthermore, we obtained new insights into the heterogeneous distribution of ionic phases by high-resolution conductivity images. The results obtained under current flow have a direct relevance for fuel cell application.

\section{Experimental}

AFM measurement.- AFM measurements were performed using a Bruker (Karlsruhe, Germany) Multimode 8 instrument in tapping mode equipped with quantitative nano-mechanical tapping (QNM, Bruker Corp.), tunneling AFM (PeakForce-TUNA, Bruker Corp.), a gastight chamber, and a Peltier heating stage. The evaluation of the force-separation curve throughout a sinusoidal path of the AFM tip during approach and retraction delivers additional information to the topography. The distribution of adhesion force, DMT ${ }^{34}$ stiffness, deformation, energy dissipation, and, with the use of a conductive tip, current can be recorded simultaneously. The current signal measured in tapping mode is averaged by a lock-in amplifier. More experimental details can be found in earlier studies. ${ }^{22,33}$

Samples. - The samples were pieces cut from ionomer membranes of Nafion NR212 with an equivalent weight of $1100 \mathrm{~g} \mathrm{eq}^{-1}$, AQUIVION PFSA E87-05S with an equivalent weight of $870 \mathrm{~g} \mathrm{eq}^{-1}$, and an experimental grade of AQUIVION PFSA membrane including PTFE reinforcement, here named Exp1, with an equivalent weight of $790 \mathrm{~g}$ $\mathrm{eq}^{-1}$. In addition, ionomer samples have been prepared from aqueous AQUIVION PFSA D79-20BS dispersion (membrane Exp2).

Preparation.-To prepare cross sections, the samples were sandwiched between two sheets of polystyrene and then cut by microtome. For AFM investigation, the samples were fixed to the sample holder by adhesive tape (Figure 2).

For the measurement of ionic current, one side of the sample was coated with a porous electrode prepared from a mixture of carbon, ionomer, and catalyst particles similar to that of a fuel cell electrode, backed by carbon paper (SGL Sigracet 25BA). This electrode layer was dried in an oven at a temperature of approximately $80^{\circ} \mathrm{C}$ or cured in-situ at the AFM heating stage. For measurement, a cross section of the whole sandwich including the two polystyrene sheets was cut

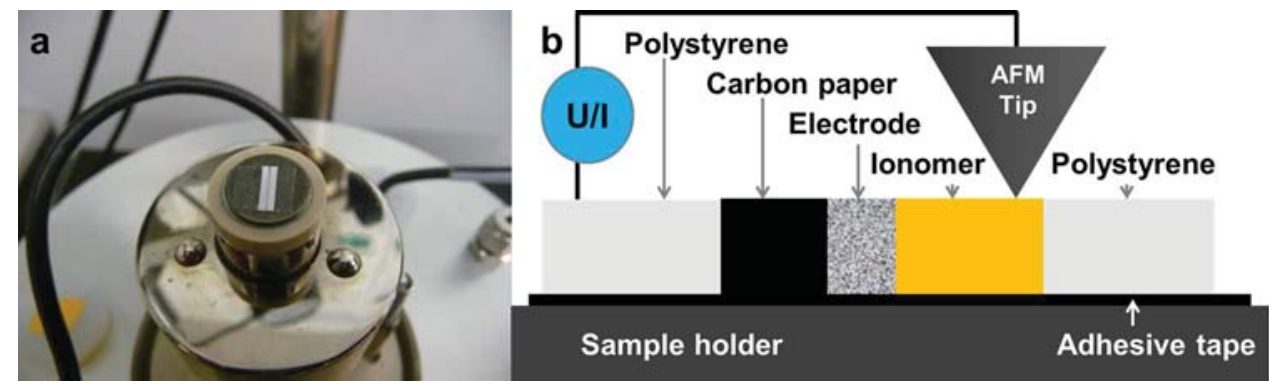

Figure 2. (a) Cross section of ionomer membrane sample on the sample holder and (b) cross section of microtome cut for current measurement. 
by microtome and glued with conductive tape or silver epoxy to the sample holder (Figure 2). For certain experiments, a back electrode was provided by a conductive layer applied to the sample holder beneath the microtome cutting. For current measurement, the top nanoelectrode was provided by the use of a catalytically active platinum coated AFM tip. If not otherwise stated, the ionomer samples were activated for $2 \mathrm{~h}$ before current measurement using an electrolysis set-up with an applied voltage of $2.8 \mathrm{~V}$ between the sample back electrode and a platinum sheet as a front contact to the bare ionomer. ${ }^{22,23}$ The back electrode was biased negatively to avoid carbon oxidation in the electrode. After the activation procedure, the water content of the samples was increased by approximately $9 \%$ (Nafion 212) determined by mass increase. Afterwards, the samples were stored in water. Most of the samples were measured in air at a relative humidity of 30-40\% and were equilibrated under these conditions. One sample was stored at elevated humidity to increase its water content prior to cutting. For a few measurements the humidity inside the AFM gastight chamber was kept constant at elevated relative humidity with an internal humidifier. The details on humidity and pretreatment are given for each Figure.

In-situ membrane curing.-The curing of AQUIVION PFSA Exp2 was performed using a heater/cooler scanner equipped with an additional Peltier element that enables heating of the sample inside the AFM up to $250^{\circ} \mathrm{C}$. The temperature of the scanner and Peltier stage were controlled by a thermal application controller. The curing of membranes followed procedures similar to the curing of commercial membranes, starting with a temperature hold for $30 \mathrm{~min}$ at $50^{\circ} \mathrm{C}$ to evaporate the solvent. Then, the temperature was increased at a rate of approximately $5^{\circ} \mathrm{C} / \mathrm{min}$ up to $190^{\circ} \mathrm{C}$ followed by a temperature hold at $190^{\circ} \mathrm{C}$ for $50 \mathrm{~min}$. Cooling was performed in air or under a drop of water. Also the back electrodes were tempered between $120^{\circ} \mathrm{C}$ and $150^{\circ} \mathrm{C}$ to obtain a superior bonding to the membrane.

\section{Results and Discussion}

Phase distribution on membrane cross sections.- At membrane surfaces in equilibrium with air, mostly hydrophobic backbonerich phase is expected, with the ionic side chains folded to the interior. $^{35,19,36,37}$ The existence of a surface skin layer with a different orientation of phase structures, ${ }^{21}$ different hydrophobicity, ${ }^{37}$ and a different degree of crystallinity, ${ }^{38}$ has been demonstrated. An investigation of the outer membrane surface may therefore not be representative of the bulk. The distribution of ionic phases has therefore been studied at freshly cut cross sections. After cutting in air the new surface of the cross section will certainly develop a new surface skin layer. After cutting, first measurements were performed after a few minutes. Surface changes during this time period could therefore not be detected. During the subsequent AFM measurements care was taken that the surface properties, mainly adhesion and stiffness, did not change, and new cuts were applied frequently to avoid aging. A detailed study of the evolution of interface properties in dependence of time after cutting will be provided separately.

First, we discuss the measured membrane properties of larger micrometer scale areas in Figure 3. Figure 3a shows the topography of a freshly cut cross section of Nafion NR212 at a scale of 10 micrometers. The surface is smooth with a roughness of only a few
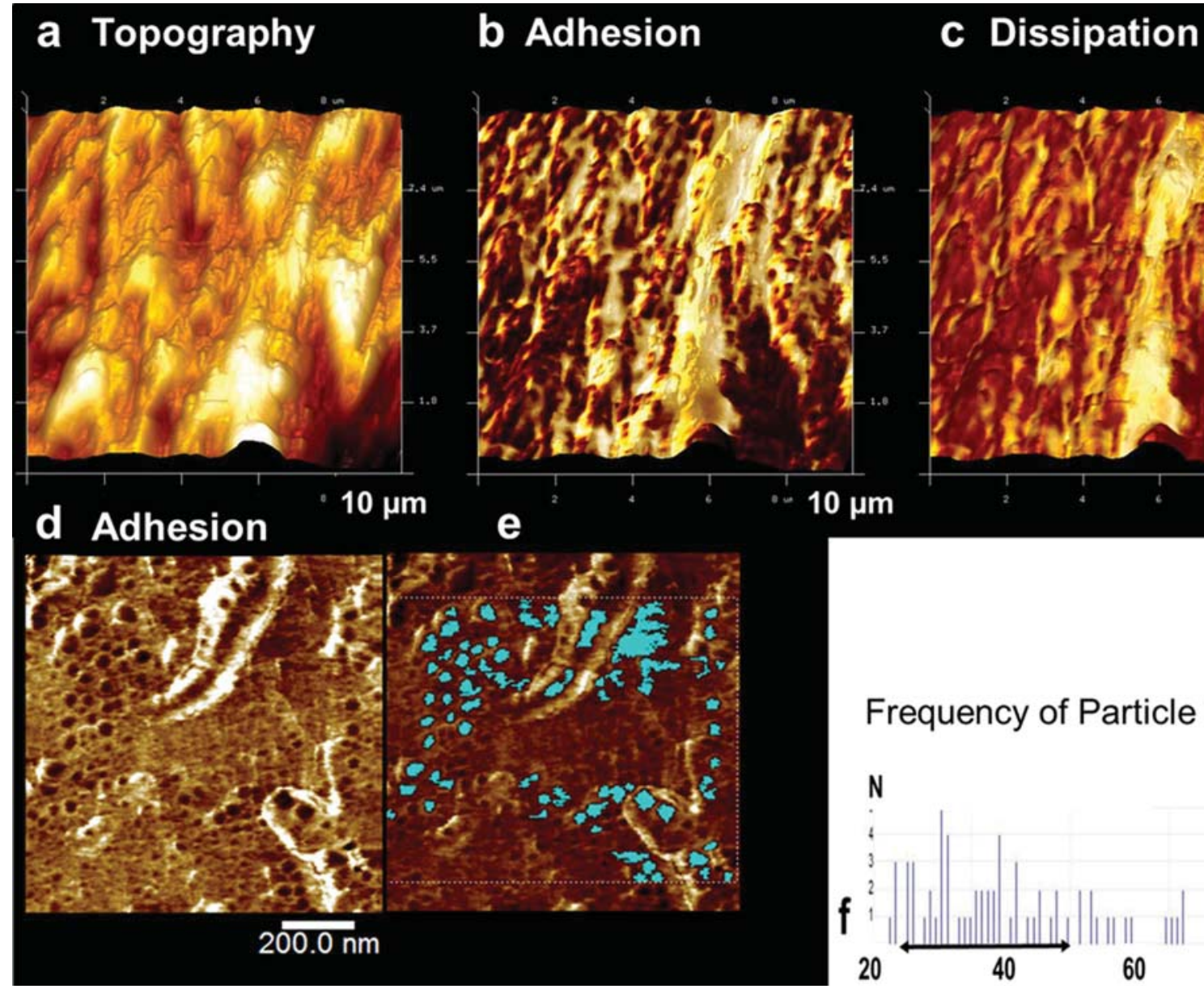

\section{Dissipation}

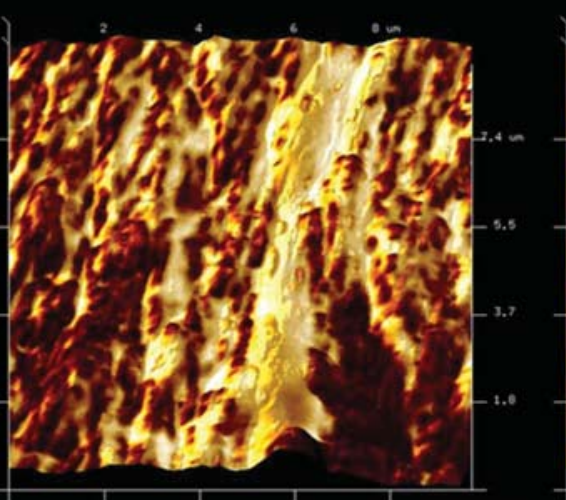

$0 \mu \mathrm{m}$

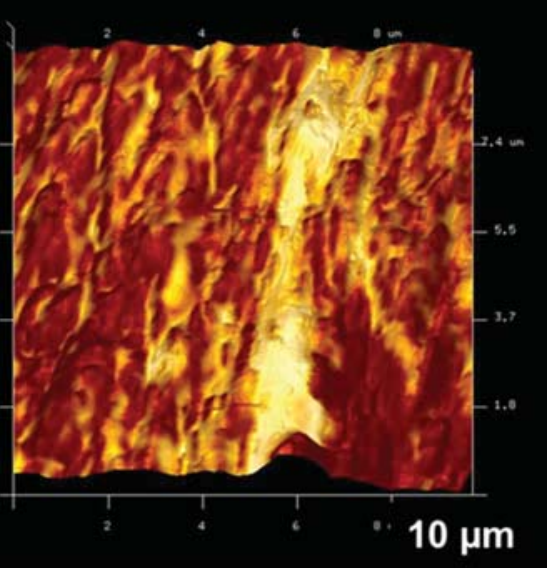

Figure 3. Material-sensitive AFM images with (a) topography, (b) adhesion, and (c) energy dissipation images of a freshly cut cross section of Nafion NR212 measured at $40 \% \mathrm{rh}$; (d) adhesion image of cross section after in-situ exposure to $70 \%$ rh for approx. 2 h, measured at $70 \%$ rh; (e) adhesion image of $d$ with low-adhesion structures marked; (f) histogram of marked areas of (e) with a mean diameter of $40 \mathrm{~nm}$. 
Nafion ${ }^{\circledR} 212$

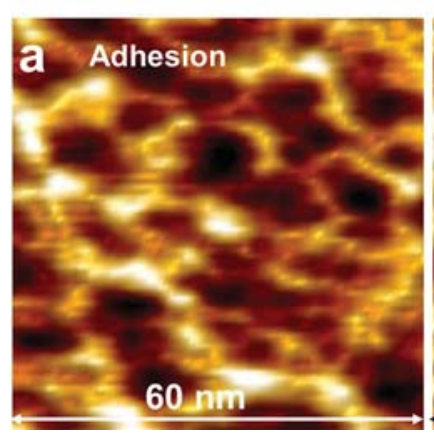

Aquivion ${ }^{\circledR}$ PFSA

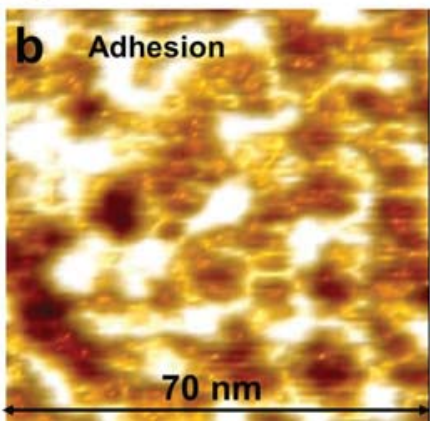

Figure 4. (a) High-resolution low force adhesion images of cross section of Nafion NR212 with a side length of $60 \mathrm{~nm}$ and (b) AQUIVION PFSA E87-05S with a side length of $70 \mathrm{~nm}$, measured at $40 \%$ rh.

ten nanometers. In the adhesion force mapping in Figure 3b, darker and brighter areas can be distinguished, but also larger bright regions can be discerned. The dissipation image in Figure $3 c$ shows essentially the same distribution with a high dissipation at areas that are high in adhesion. The adhesion force is restricted to the surface from where the signal is measured, but deformation and energy dissipation are also influenced by the sub-surface properties of a small volume beneath that is deformed by the impression of the tip. It is evident that two different phases with a significant variation of mechanical properties exist. It should be noted, that nanoscale ionic phase must be present everywhere in the sample. At locally increased water content merging of these ionic regions may form larger water-rich areas and if connected a continuous ionic network that can support current flow forms. In our previous studies, ${ }^{22,23}$ the regions that had a low adhesion, were attributed to such an interconnected ionic phase, and regions with higher adhesion were assigned to the non-conductive phase. The observation of lower deformability of water-filled interconnected ionic phase in in agreement with the reported high internal pressure in the ionic network. ${ }^{21,39}$ In the previous investigations we always found high adhesion on PTFE containing surfaces in twomaterial composites. On a smaller scale image, it becomes apparent that the large low adhesive areas have a substructure with circular water-filled ionic regions, induced by the surface tension. They have a size averaging $40 \mathrm{~nm}$, evaluated by the NanoScope Analysis software (Bruker, Version 1.50) (see Figures 3d and 3e). Diameters of 30 to 50 $\mathrm{nm}$ are typically measured for water-filled ionic phase structures on this scale range (see histogram in Figure 3f). These $40 \mathrm{~nm}$ water-filled ionic areas are obviously not the primary ionic clusters reported in the literature, which are assumed to have a size of a few nanometers. A structural size of $50 \mathrm{~nm}$ has been reported by Gebel et al. for a unit of aligned bundles. ${ }^{10,40}$ However, he assumes these are aligned backbone bundles. In our case the water-filled ionic regions are assumed to be amorphous.

Next, we discuss the distribution of water-filled ionic areas on a smaller scale, measured with low force and an especially sharp tip (BudgetSensors SHR150) to minimize distortion of the surface. Figures $4 \mathrm{a}$ and $4 \mathrm{~b}$ show the adhesion images of Nafion NR212 and AQUIVION PFSA E87-05S with nanometer-sized structures. It is evident that smaller ionic phases (dark) exist in smaller areas, which could be interpreted as the primary ionic clusters. In the AQUIVION PFSA ionomer, the size of the backbone phase (bright) is slightly larger. However, we note that due to the high-resolution experimental procedure, the top-most surface layer has most likely already experienced a modification induced by loss of water.

Morphology of membrane cross sections. - The topography images of freshly cut cross sections show terraced surfaces over several micrometers. The terraces are separated by numerous small steps with varying heights. In Figures $5 \mathrm{a}$ and $5 \mathrm{~b}$, the cross section of Nafion NR212 stored at $30 \%$ rh with a side length of $2 \mu \mathrm{m}$ is imaged. The measurements were performed in ambient air with a relative humidity of approximately $30 \%$. The 3D-topography in Figure $5 \mathrm{a}$ is characterized by an overall highly stepped surface. In the corresponding adhesion mapping overlaid on the 3D-topography data (Figure 5b), the distribution of embedded low-adhesive water-filled ionic phase is visible. The topography image gives no indication on the location of the water-filled ionic phase. These circular water-filled ionic areas that appear dark in the adhesion image measure approximately 30 to 50 $\mathrm{nm}$ in diameter, similar to the previous results. The sample of which a freshly cut cross section is pictured in Figure 5c was stored at $75 \%$ rh for 2 days with a subsequent water uptake. The topography image shows a few almost flat terraces extended curved structures that can be interpreted as sheets or stacked with lamellar. The lamellar have widths of 3 to $20 \mathrm{~nm}$ and heights of 1 to $5 \mathrm{~nm}$. Often, the curved lamellar form onion-like structures that enclose a terrace-like core oriented in different directions. The lamellae represent an ordering of the polymer structures in the range of micrometers and dominate the images. These are not the crystalline areas reported to comprise approximately $20 \mathrm{vol} \% .^{2}$ According to the literature, these crystallites have a size in the range of a few nanometers. ${ }^{3}$

Since we observe ordered domains in approximately $80 \%$ of the area they must be associated with both, backbone and ionic parts Over time the observed ordered lamellae disappear in contact with air. We can exclude that the cutting process has induced the ordering because we observed highly stepped surfaces without cutting from casted membrane interfaces solidified in contact with water. As shown in Figure $5 \mathrm{c}$, the stacking direction of the lamellae is changing significantly, which could lead to an overall amorphous signal in scattering experiments. In only very few freshly cut samples, no steps were observed, which may be caused by inferior tip sharpness. In the literature, some authors already proposed a lamellar stacking in Nafion, ${ }^{14,15}$ however, such a structure has not been directly observed. Recent models assume a high degree of disorder ${ }^{17}$ and do not seem to include micrometer larg regions with such high degree of ordering. After extended exposure of the interface to ambient air, it was no longer possible to observe lamellae or terraces and no such structures have ever been imaged at membrane outer surfaces in equilibrium with ambient air. In contrast, the surface cooled under water after curing is highly ordered with numerous terraces, as visible in Figure $5 \mathrm{~d}$ and in the corresponding peak force signal that enhances the terrace edges in Figure 5e. In conclusion, we deduce that the lamellar structures are characteristic of the bulk ionomer but relax upon exposure to air.

In order to analyze the height distribution of the lamellae the terrace-like areas equilibrated and measured at $30 \%$ rh were investigated. Before measurement of the height value of one layer at the lamellar edge their perpendicular orientation was first clarified from topography images. The layer height of the thinner lamellae has been measured on cross sections of a cut from Nafion NR212 membrane and from AQUIVION PFSA E87-05S. Approximately 180 layers were evaluated each by using the NanoScope Analysis software. In order to ensure a correct layer height of the lamellae, all steps in the profile were manually oriented vertically with the software before the value was taken. The measurement of vertical heights with AFM is not much affected by convolution of tip shape and therefore quite precise. To ensure a superior height resolution, the maximum voltage of the z-height channel of the AFM was reduced. Both histograms of the measured lamellar heights on Nafion NR212 and AQUIVION PFSA E87-05S given in Figure 6 reveal a wide distribution with specific accumulation points that indicate the most probable heights of layers. We also found larger layers that were not included in this evaluation. The observed large variation in lamellar thickness is consistent with an interpretation of embedded water sheets. It is assumed that the highest peak in the histograms may be associated with the most stable layer width. Both histograms show a comparable sequence of peaks at certain step heights, but in the case of AQUIVION PFSA, the position of all peaks is always shifted toward a smaller layer thickness. The AQUIVION PFSA ionomer consists of molecules with shorter side chains, which is clearly reflected in the histogram. The highest peak and therefore the most probable lamellar thickness of Nafion were 

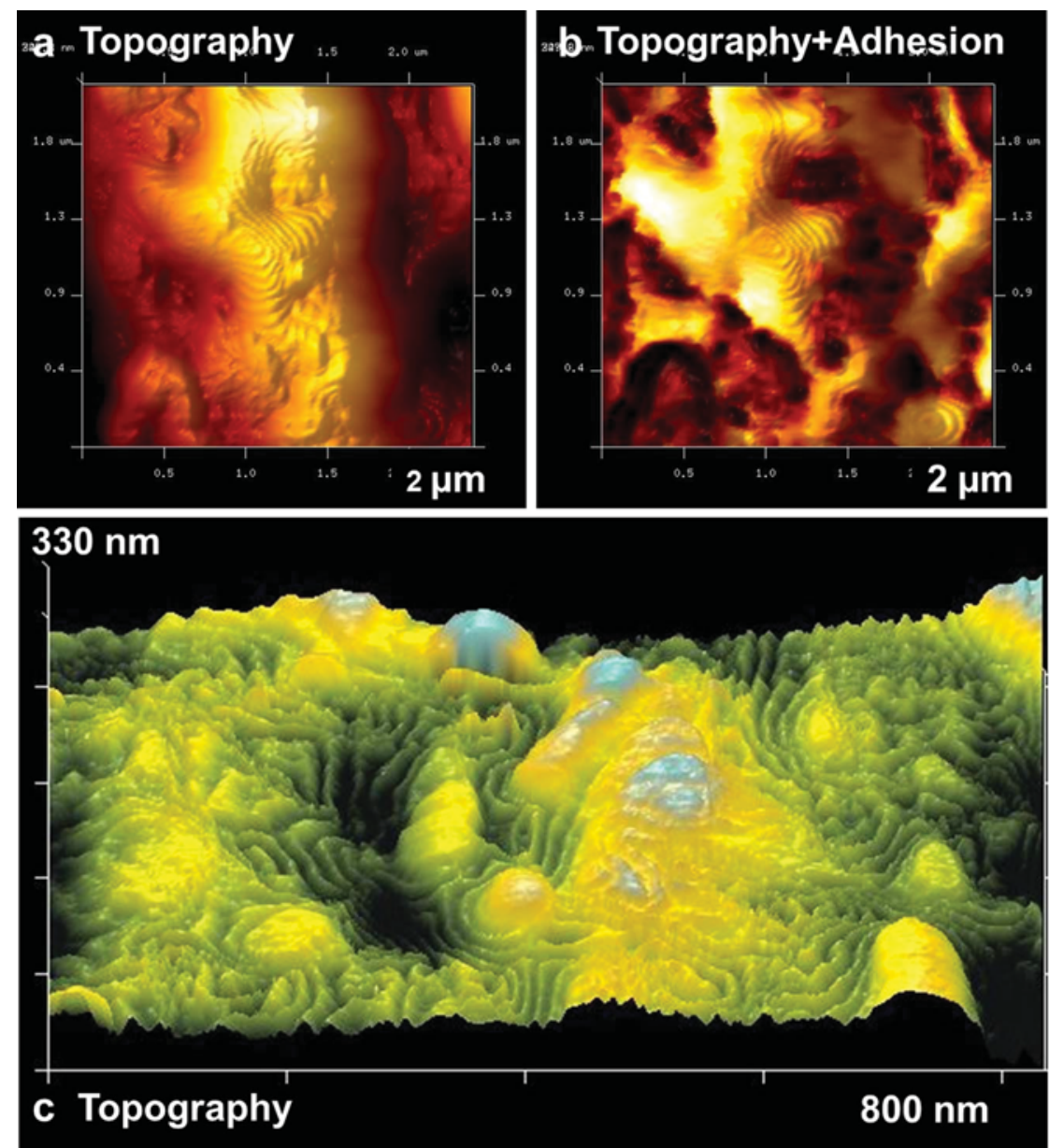

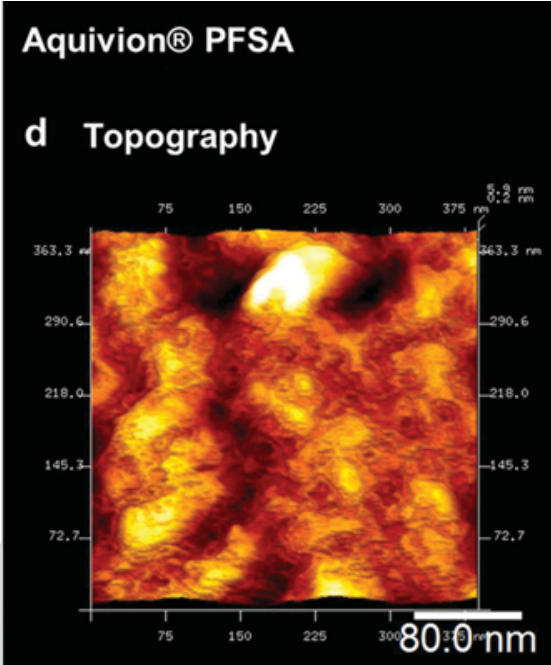

e Peak Force

d

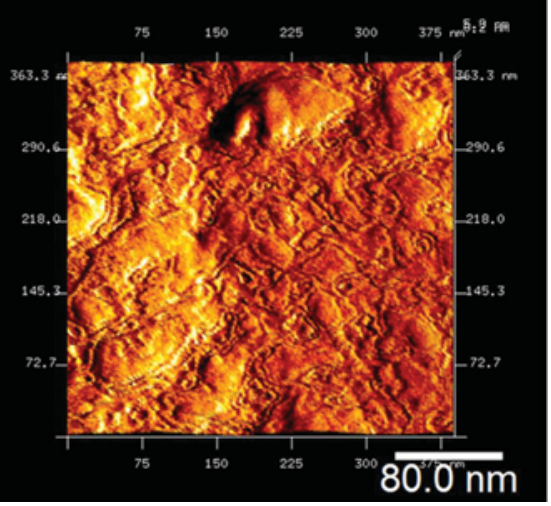

Figure 5. AFM 3D-topography images of a freshly cut cross sections of Nafion NR212 in lamellar regions: (a) topography and (b) corresponding adhesion images with distribution of ionic phase measured at 30\% rh; (c) highly stepped lamellar structure after storage for 2 days at $75 \%$ rh, measured at $40 \%$ rh; (d) membrane surface topography of AQUIVION PFSA Exp2 equilibrium interface with water, measured at $40 \% \mathrm{rh}$; (e) corresponding peak force signal with enhanced step edges.
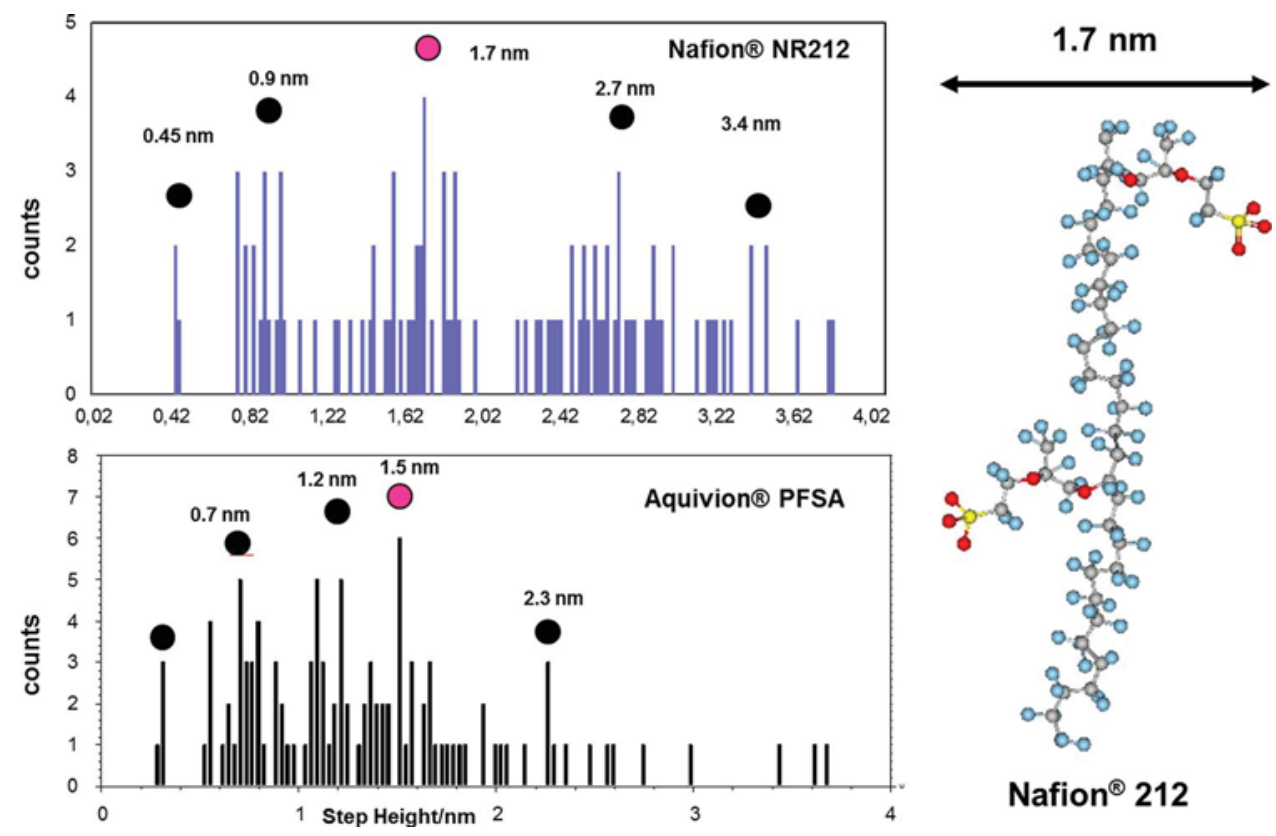

Nafion $^{\circledR} 212$

Figure 6. Histograms of measured step heights on ordered regions of freshly cut cross sections of Nafion NR212 and AQUIVION PFSA E87-05S samples measured at $30 \% \mathrm{rh}$. At right: illustration of the size of one Nafion polymer backbone strand with two side chains. 
$1.7 \mathrm{~nm}$ and $1.5 \mathrm{~nm}$ of AQUIVION PFSA. The estimated width of one molecular backbone and two side chains with half their outstretched size (comp. Figure 6 on the right side) corresponds to the width of two backbone chains with two side chains in-between, namely $1.7 \mathrm{~nm}$ and $1.5 \mathrm{~nm}$ for Nafion and AQUIVION, respectively. The smallest layer thickness would fit the length of a single (folded) side chain. The solvated hydrophilic sulfonic acid groups at the end of the side chains form a two-dimensional sheet-like ionic conductive network for water transport in correspondence to Figure $5 \mathrm{c}$, in which a composition of the polymer from lamellar sheets is visible. The wide distribution of lamellar thickness indicates that the amount of embedded water can vary significantly dependent on the conditions. This variance also explains the different morphologies in Figures $5 \mathrm{a}$ and $5 \mathrm{c}$.

Conductive structure of Nafion and AQUIVION PFSA.Capacitive current at cross section.-With conductive AFM we measure two different current contributions. When the AFM tip approaches the proton conducting electrolyte surface an electrochemical double-layer is formed. Therefore both, scanning the tip across the membrane and tapping toward the surface, will give rise to a capacitive current according to the differential capacitance and the scan rate. The $i(V)$ curve recorded at a wet Nafion 212 surface in air at approx. $40 \%$ rh shows the current measured by the AFM tip (precisely, the current density for given constant tip area) for a voltage scan. As known from such voltamograms, a capacitive current contribution that results from charging of the electrochemical double layer leads to a hysteresis of measured current values in the two scan directions. For a surface charging, the dependence of the capacitive current contribution, which is proportional to the separation of the two curves, marked with an arrow in Figure 7a, varies linearly with scan rate. This observed dependence is depicted in Figure $7 \mathrm{~b}$. In case of electrochemical reaction additional current peaks show up at their specific potential. According to these $i(V)$ curves which show the presence of a double layer, a locally higher capacitive current can be attributed to a higher charge density and water content at the ionomer surface.

The typically small current due to the local capacitance of the ionomer interface can be used to monitor the distribution of waterrich and water-poor regions. In contrast, the larger scale continuous current, which is relevant for the application, relies on highly waterfilled interconnected networks between the two electrodes. A continuous current is associated with faradaic reactions and suitable electrode potentials. The small capacitive current persists even if a larger current flow in the range of $\mathrm{nA}$ is present at the same time after activation. ${ }^{22}$ Due to the large difference in current magnitude, the capacitive currents are not discerned when continuous reaction dependent currents are measured but are evident in current histograms.
In Figure 8 a freshly cut cross section of Nafion NR212 is shown in two magnifications with topography, adhesion force, and capacitive current images. Here, the topography images show a terraced surface composed of lamellar layers with some elevations that expose lamellar edges at their slopes. These lamellar edges indicate the ends of layers that surround the hills. The corresponding adhesion images show that these hills have somewhat lower adhesion that corresponds to some water-filled interconnected ionic phase. We assume that the elevations are the consequence of local swelling due to hydrostatic pressure. The distribution of capacitive current is more complicated. Higher capacitive currents indicate an ionic phase with high proton density and high water content. The higher conductive regions form rings around the hills, following the assumed layers. Along the rings, which have a thickness of a few nanometers, the conductivity is heterogeneous, with spots of higher and lower current. In certain regions, bands with extremely low current values are visible that could indicate backbone strands without water. It can be concluded that both, water-rich layers and water-poor layers are present. This description is in agreement with the assumption of water-filled sheets. Ionic regions formed by solvated sulfonate end groups of the side chains are enclosed between backbone layers. They contain the water and the associated protons to provide conductivity. These observations support nanostructure models that propose water-filled lamella, as deduced already in 1980 from infrared measurements and the linear dependence of structure size on water content ${ }^{13}$ and recently in addition from electrostatic considerations. ${ }^{21}$

In Figure 9, adhesion and capacitive current images of an area of $(325 \times 325) \mathrm{nm}^{2}$ of a freshly cut cross section of AQUIVION PFSA E87-05S membrane are shown. Here, the current is also very low, with a magnitude of a few pA. The low adhesion area visible on the left corresponds to the higher conductivity phase on the right side. The conductive low adhesion phase in the conductivity image is surrounded by dark structures with lower proton/water content. Qualitatively, the same interpretation derived for Nafion, namely, the high current areas identified as conductive layers, is applicable also to AQUIVION PFSA.

A direct comparison of the conductivity structure in the capacitive current distribution of cross sections of Nafion NR212, AQUIVION PFSA E87-05S, and AQUIVION PFSA Exp1 membranes is shown in Figure 10 for an area of $1 \mu^{2}$. The comparison shows that the distribution of water-rich ionic phase is less pronounced for the AQUIVION PFSA E87-05S, which has much larger connected conductive regions. This result is in agreement with molecular dynamics studies in the literature ${ }^{18}$ where higher connectivity of conductive areas was calculated for the short side chain type of molecule.

The total percentage of conductive areas on the whole surface does not differ significantly between Nafion NR212, (34 \pm 3$) \%$,
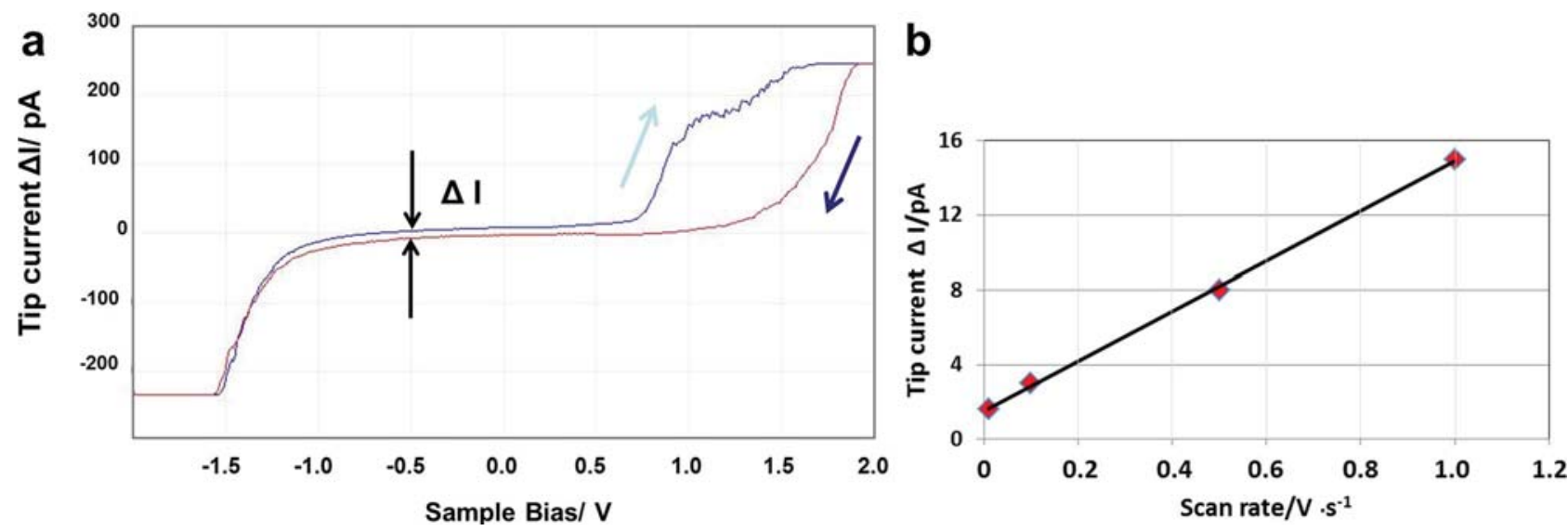

Figure 7. (a) i(v) curve measured at wet Nafion 212 at air with constant tip area, (b) Dependence on current hysteresis $\Delta \mathrm{i}$ as a measure for the capacitive current with varying scan rate. 


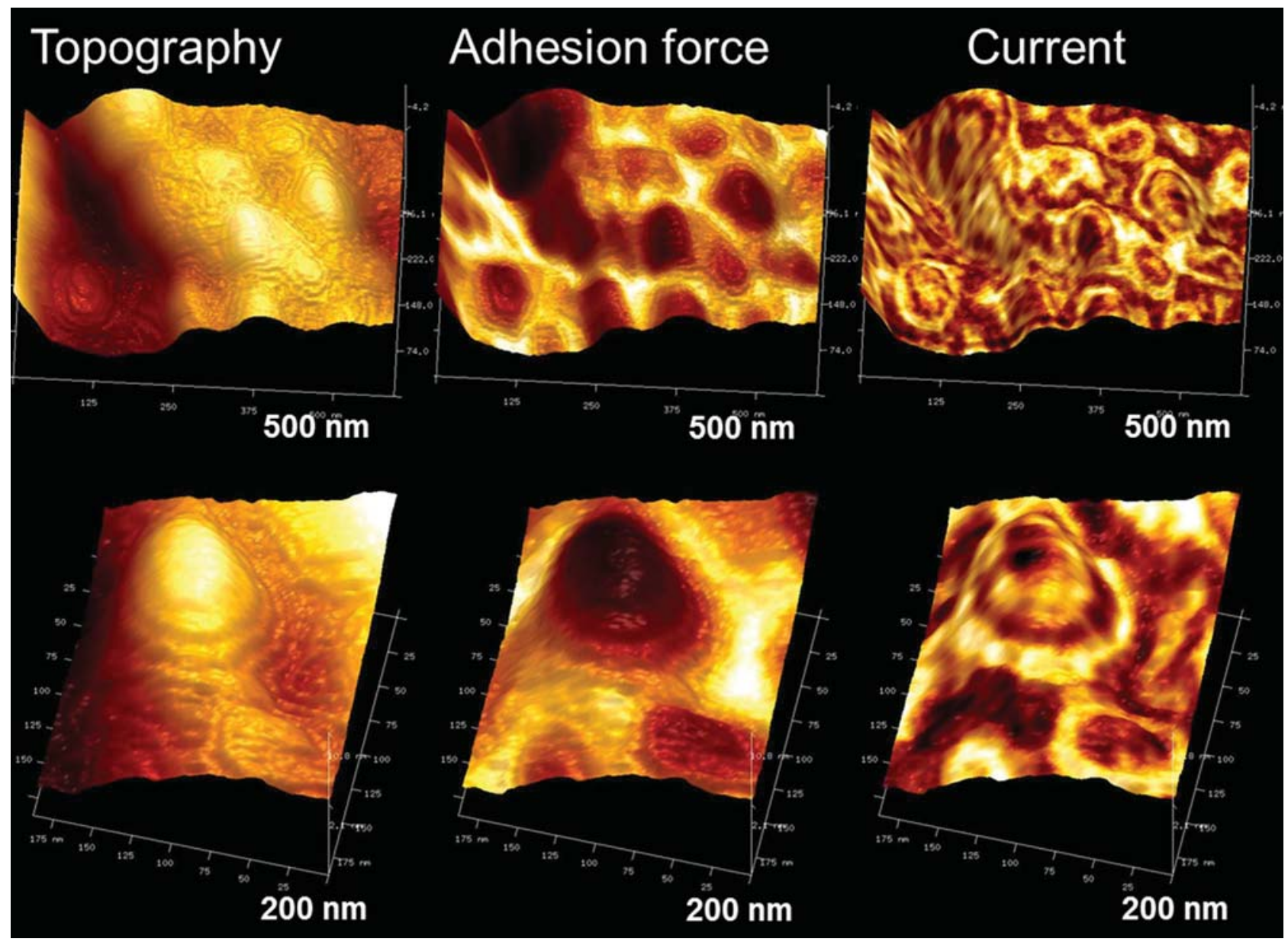

Figure 8. AFM images showing topography, adhesion force, and capacitive current at lower (top row) and higher (bottom row) magnification of a freshly cut cross section of Nafion NR212 measured at $40 \%$ rh.

AQUIVION PFSA E87-05S, $(38 \pm 3) \%$, and AQUIVION PFSA Exp2, $(34 \pm 3) \%$. The values are given with the mean error of the mean for one standard deviation.

Another indication for the existence of conductive layers can be observed in the image sequence in Figure 11. Here, two subsequent measurements on a cross section of Nafion NR212 show a different distribution of conductive area of capacitive current. A small drift leads to a shift in structures with time and to a small elliptical distortion of the structures. The capacitive current that indicates the existence of water-rich areas was in the range of a few $\mathrm{pA}$. Alternating layers with high and low conductivity indicate an onion-like stacking of layers of different conductivity with layers oriented differently to the surface (as also visible in Figure 10). Comparison of the encircled areas of Figures $11 \mathrm{a}$ and $11 \mathrm{~b}$ show the removal of the conductive top layer by larger applied forces of the AFM tip. The bright, highly conductive top, visible in Figure 11a, is followed by a dark low-conductive phase, visible in Figure 11b. However, the remaining slightly changed conductive features can still be recognized in Figure 11a, showing that the vertically oriented conductive layers remain and propagate into the bulk. In Figure $11 \mathrm{c}$, a high-resolution image of one conductive
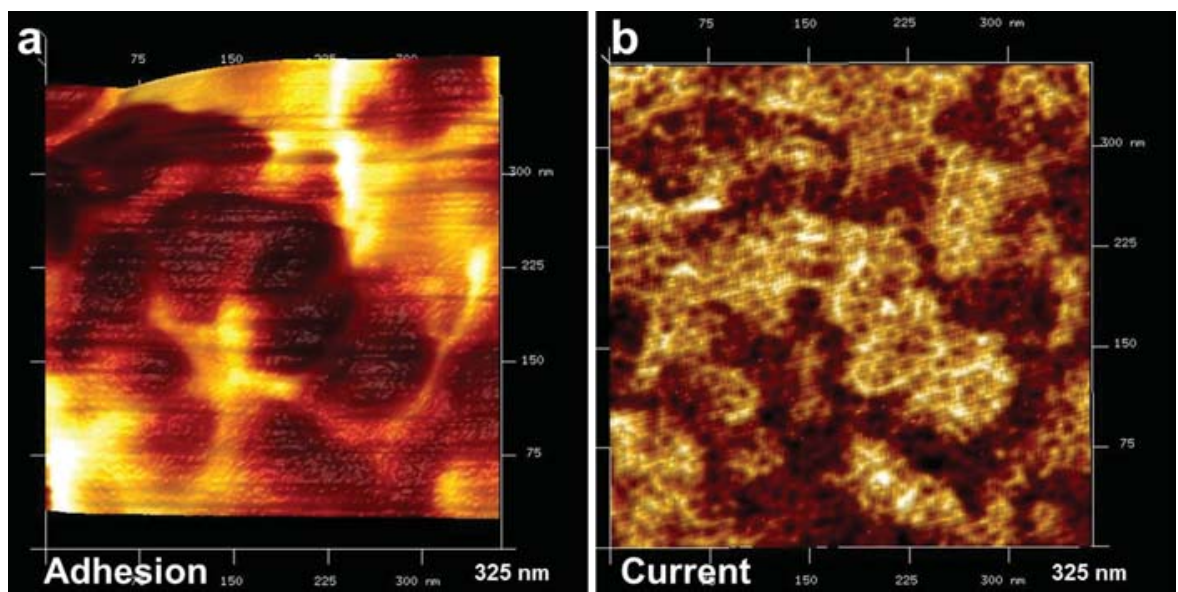

Figure 9. AFM images of freshly cut cross section of AQUIVION PFSA E87-05S with (a) distribution of adhesion forces and (b) current measured at $40 \%$ rh. 


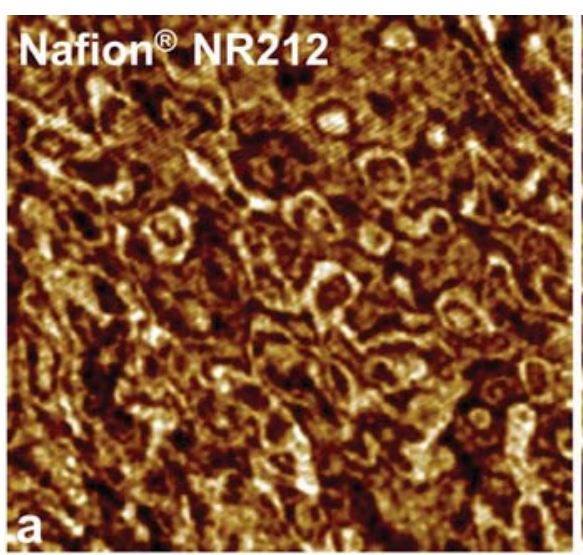

Current

$\Delta \mathrm{l}=\mathbf{5 0} \mathrm{pA}$

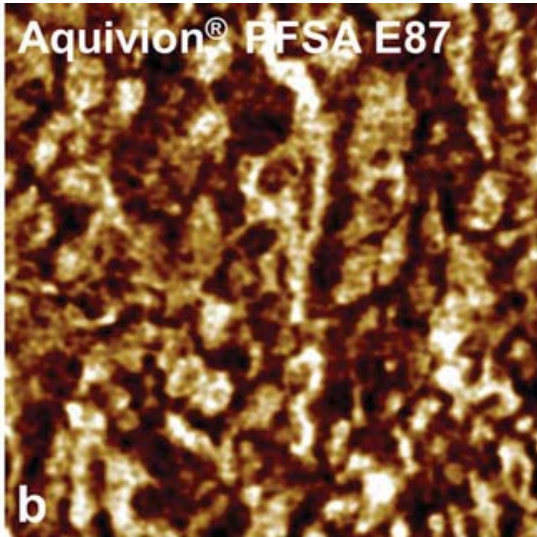

Current $\quad \Delta \mathrm{l}=70 \mathrm{pA}$

$1 \mu \mathrm{m}$

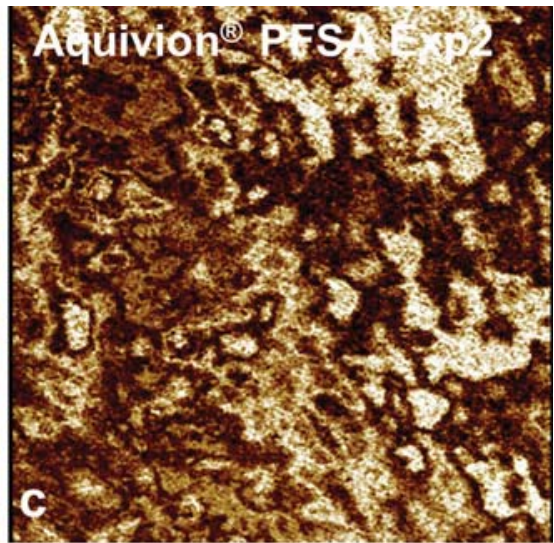

Current

$\Delta \mathrm{l}=1 \mathrm{pA}$

$1 \mu \mathrm{m}$

Figure 10. AFM images of cross section of capacitive current on (a) Nafion NR212, (b) AQUIVION PFSA E87-05S, and (c) AQUIVION PFSA Exp2 samples measured at $40 \% \mathrm{rh}$

ring is shown. Although the current of the conductive ring is small, a modulation of the current magnitude along the conductive layer is present. The thickness of the conductive layers is in the range of 7-10 $\mathrm{nm}$ for the smallest structure and up to $50 \mathrm{~nm}$ for larger area structures. The brighter spots on the ring are approximately $4-6 \mathrm{~nm}$ wide, as shown in the high-resolution image in Figure 11c, which gives an upper limit for their size.

Steady state current at cross section. - In technical applications, a continuous current through PFSA is necessary. However, at a freshly cut cross section of non-activated Nafion NR212, typically only small capacitive currents with a magnitude of a few pA or less were measured. After approximately $1 \mathrm{~h}$ of scanning under applied voltage in a humid environment, much higher continuous currents in the range of several nA were observed in certain areas. This activation process is always observed ${ }^{22}$ and can be induced also by forcing currents through the membrane. The proton flow is accompanied by water due to the electro-osmotic drag and on average transports $2-3$ water molecules per proton. The initially small current thereby exerts mechanical pressure on the ionic phase, which leads to structural changes. The ionic clusters merge, followed by increased current/water flow through the sample. ${ }^{33}$ It is likely that no continuous conductive network exists before activation but is formed only under current flow. Another factor that influences the current flow is the fast formation of a dry thin surface skin layer of lower conductivity after exposure of the ionomer to air. ${ }^{18}$ The orientation change of ionic end groups at the surface is assumed to last only a few seconds and is driven by the immediate loss of water activity at the interface. An initial current flow then leads to re-wetting of the surface and induces a change to a more hydrophilic surface with open ionic pores. It is likely that this skin layer formed at the cross section is initially very thin and grows over time.
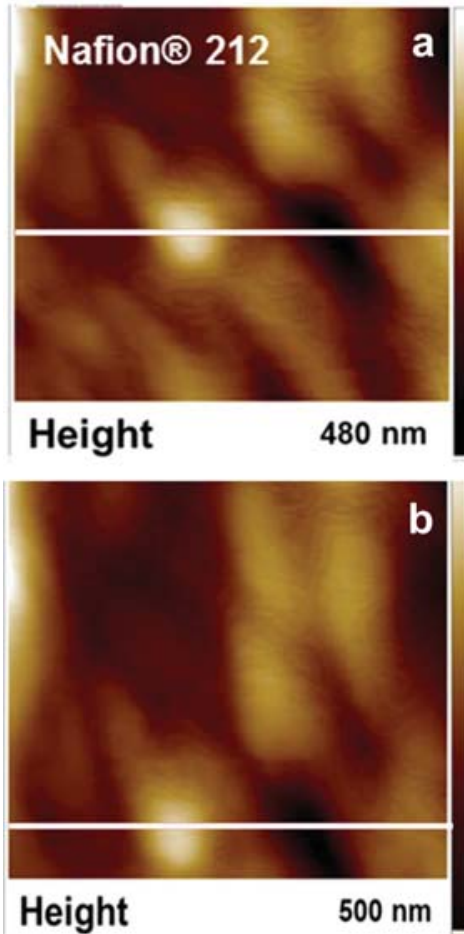
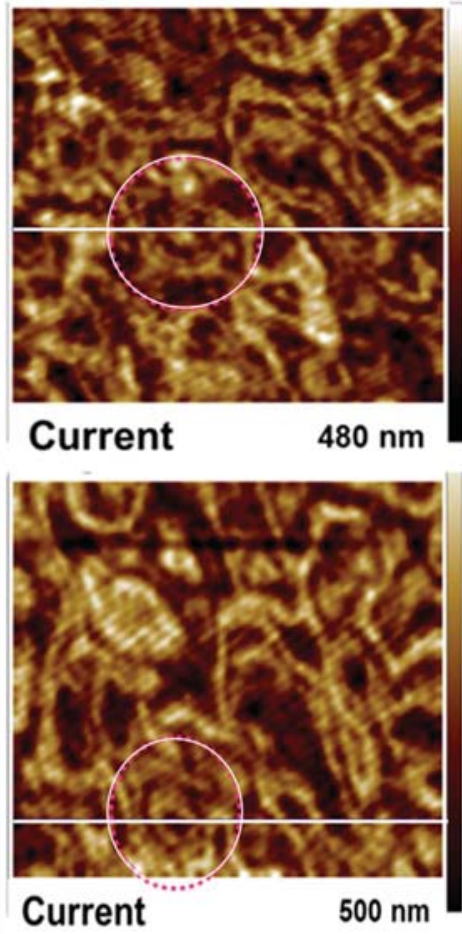
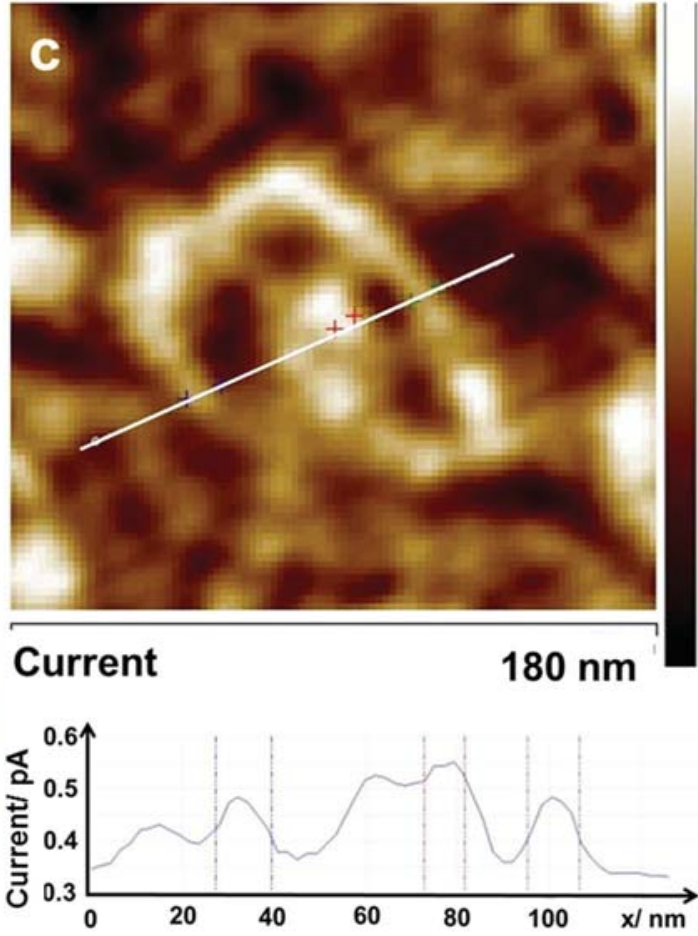

Figure 11. (a, b) Two subsequent conductivity images of a freshly cut cross section of Nafion NR212 with a circle around the same spot; (c) current profile along the marked line in the image measured at $40 \%$ rh. 


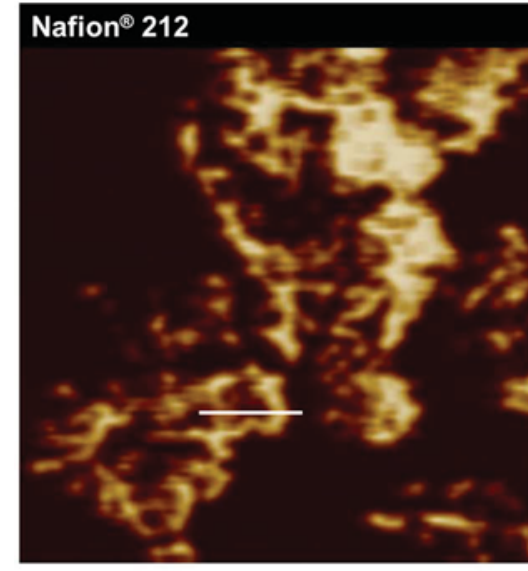

a Current

$500 \mathrm{~nm} \Delta \mathrm{l}=5 \mathrm{nA}$
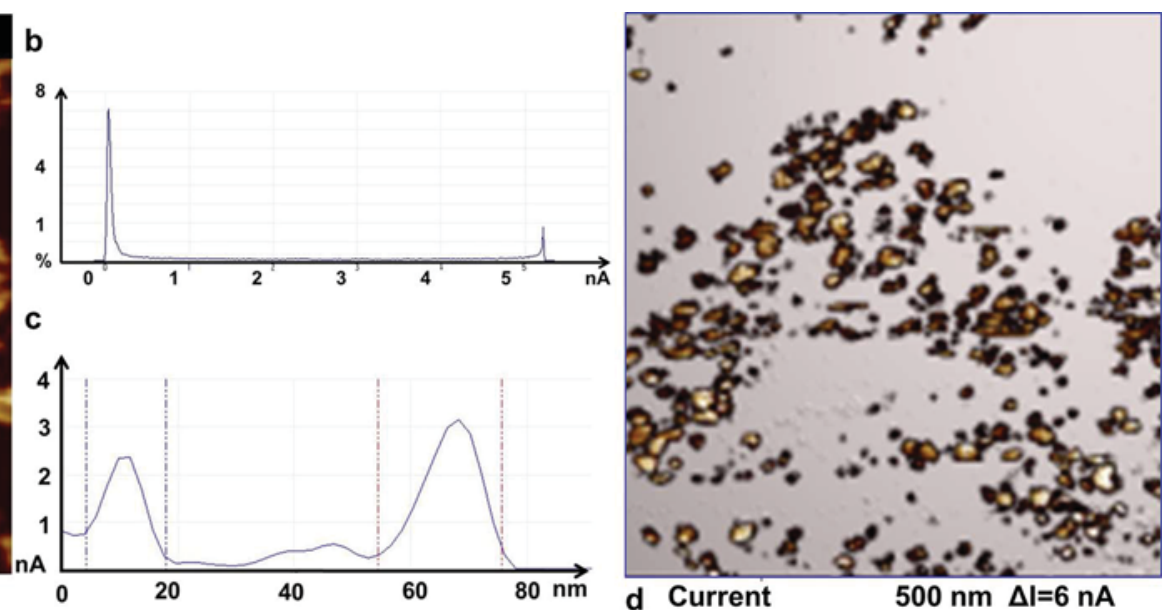

Figure 12. (a) Steady-state current image of a freshly cut cross section of Nafion NR212 measured at $40 \%$ rh; (b) histogram of the relative current distribution across the whole scanned area; (c) current profile across a larger conductive area, marked in 12a; (d) steady-state current distribution on activated outer membrane surface with skin layer measured at $40 \% \mathrm{rh}$.

Figure 12a shows the current distribution of an activated Nafion NR212 membrane cross section with heterogeneously distributed current areas with a current in the range of nA. The dark areas between the higher conductive bright areas correspond to lower current densities, as shown in the current profile (Figure 12c) of a line scan marked in Figure 12a. A histogram of the total current in Figure 12b shows the bimodal current distribution with a capacitive part in the $\mathrm{pA}$ range and a higher faradaic current contribution of several nA. Only a small percentage of the area is highly conductive, ${ }^{31}$ with an average current of $5.4 \mathrm{nA}$. On a larger scale, the total lateral size of the conductive areas in Figure 12a is between $30 \mathrm{~nm}$ and $50 \mathrm{~nm}$, the total size is approximately $200 \mathrm{~nm}$. The smallest visible features were in the range of two nanometers, which is comparable to the distance of two backbone layers with two side chains in-between as proposed from the measurement of the lamella heights (Figure 6).

The observed current areas are mostly interconnected and are therefore not consistent with the parallel nanochannels proposed by Schmidt-Rohr and Chan. ${ }^{16}$ These proposed parallel water channels were thought to be enclosed by molecular backbone, which is not the case here. In comparison with the onion-like structures seen in Figures 5 and 10, it is clear that these structures can be identified as cross sections of folded conductive lamellae and enclosed water-filled ionic regions. For comparison, in Figure 12d, the distribution of current measured at an outer membrane surface of Nafion 112 is shown. The current distribution of the membrane with a skin layer is more dispersed, with isolated current spots. In contrast to the measurements of cross sections, the current is zero between the spots. This observation results from the hydrophobic surface layer, which has only a few defects.

\section{Conclusions}

AFM investigations of cross sections show two distinct phases that differ in their mechanical properties. The phase of lower adhesion forms mostly circular areas of 30 to $50 \mathrm{~nm}$ with smaller substructures in between. It is attributed to water-filled interconnected ionic phases. The area with high adhesion is associated with the hydrophobic backbone-rich phase and non-connected water-poor ionic phase.

At freshly cut cross sections of all investigated PFSA membranes, large micrometer-wide areas that exhibit highly ordered lamellae are visible. The layer thickness distribution is wide, but in a histogram, their preferential layer thicknesses are significantly smaller in AQUIVION PFSA than in Nafion PFSA and are comparable to the length of the specific molecular side chains, indicating stacked layers of back- bones and ionic side chains in-between. These ordered regions inside the bulk are the first direct imaging of the proposed lamellar structures for Nafion. The bent layers form onion-like structures often enclosing a core and are randomly oriented.

The current measured by tapping mode AFM consists always of a capacitive contribution with small currents of a few pA that reflect locally high water content. The high-resolution conductivity images of these capacitive currents show the size and distribution of the ionic phase ranging in size from 3 to approximately $10 \mathrm{~nm}$. A comparison of Nafion NR212 and AQUIVION PFSA E87 demonstrates larger water-rich connected regions in AQUIVION PFSA, although the total conductive area is similar.

After activation by current flow or by AFM scanning, a continuous current on the order of a few $\mathrm{nA}$ appears. In this case a continuous ionic conductive network has formed enhanced by the electro-osmotic drag of water and a buildup of hydrostatic pressure, which determines the resulting conductive structure of the membrane. The current-induced highly conductive areas are approximately 30 to $50 \mathrm{~nm}$ in diameter, in agreement with the adhesion measurements. The comparison of the current distribution of cross sections and surfaces exposed to air reveals distinct differences. The high current areas are more distributed at the membrane surface with a skin layer, and the capacitive currents are completely absent. This observation indicates the changed properties due to the dry hydrophobic skin layer.

In our interpretation of the AFM results a bi-continuous network is present under application conditions consisting of an almost continuous phase with ionic and lamellar backbone structures and a separated network of water-filled interconnected ionic phase that provides the macroscopic conductivity. This picture differs from the recently reported model from Elliot et al. due to our observation of a dominant lamellar structure and a remaining ionic fraction in the matrix phase. ${ }^{17}$

\section{Acknowledgments}

The research leading to these results has received funding from the European Union's Seventh Framework Program (FP7/2007-2013) for the Fuel Cells and Hydrogen Joint Technology Initiative under grant agreement $\mathrm{n}^{\circ}$ 303452, "IMPACT - Improved Lifetime of Automotive Application Fuel Cells with ultra-low Pt-loading." We gratefully acknowledge technical support by Bruker Corporation in Karlsruhe.

\section{References}

1. S. J. Paddison and R. Paul, Phys. Chem. Chem. Phys., 4, 1158 (2002).

2. K. A. Mauritz and R. B. Moore, Chem. Rev., 104, 4535 (2004).

3. M. Ludvigsson, J. Lindgren, and J. Tegenfeldt, J. Electrochem. Soc., 147, 1303 (2000). 
4. K.-D. Kreuer and G. Portale, Adv. Funct. Mater., 23, 5390 (2013).

5. N. J. Economou, J. R. O'Dea, T. B. McConnaughy, and S. K. Buratto, RSC Adv., 3, 19525 (2013)

6. S. Subianto, M. Pica, M. Casciola, P. Cojocaru, L. Merlo, G. Hards, and D. J. Jones, J. Power Sources, 233, 216 (2013).

7. T. D. Gierke, G. E. Munn, and Fc. Wilson, J. Polym. Sci. Polym. Phys. Ed., 19, 1687 (1981).

8. A. Eisenberg, Macromolecules, 3, 147 (1970).

9. G. Alberti, R. Narducci, and M. Sganappa, J. Power Sources, 178, 575 (2008)

10. G. Gebel, Polymer, 41, 5829 (2000).

11. L. Rubatat, G. Gebel, and O. Diat, Macromolecules, 37, 7772 (2004).

12. L. Rubatat, A. L. Rollet, G. Gebel, and O. Diat, Macromolecules, 35, 4050 (2002).

13. M. Falk, Can. J. Chem., 58, 1495 (1980).

14. M. H. Litt, Polymer Preprints, 38, 40 (1997)

15. H.-G. Haubold, T. Vad, H. Jungbluth, and P. Hiller, Electrochimica Acta, 46, 1559 (2001).

16. K. Schmidt-Rohr and Q. Chen, Nat Mater, 7, 75 (2008).

17. J. A. Elliott, D. Wu, S. J. Paddison, and R. B. Moore, Soft Matter, 7, 6820 (2011).

18. M. Bass, A. Berman, A. Singh, O. Konovalov, and V. Freger, J. Phys. Chem. B, 114, 3784 (2010).

19. M. Bass, A. Berman, A. Singh, O. Konovalov, and V. Freger, Macromolecules, 44, 2893 (2011).

20. J. Tang, W. Yuan, J. Zhang, H. Li, and Y. Zhang, RSC Adv., 3, 8947 (2013)

21. K.-D. Kreuer, Solid State Ionics, 252, 93 (2013).

22. R. Hiesgen, S. Helmly, I. Galm, T. Morawietz, M. Handl, and K.A. Friedrich, Membranes, $2,783(2012)$

23. R. Hiesgen, E. Aleksandrova, G. Meichsner, I. Wehl, E. Roduner, and K.A. Friedrich, Electrochimica Acta, 55, 423 (2009).
24. S. Matsumura, A. R. Hlil, C. Lepiller, J. Gaudet, D. Guay, Z. Shi, S. Holdcroft, and A. S. Hay, Macromolecules, 41, 281 (2008).

25. R. Maoz, S. R. Cohen, and J. Sagiv, Adv. Mater, 11, 55 (1999).

26. Z. Wang, H. Tang, J. Li, Y. Zeng, L. Chen, and M. Pan, J. Power Sources, 256, 383 (2014).

27. D. A. Bussian, J. R. O’Dea, H. Metiu, and S. K. Buratto, Nano Lett., 7, 227 (2007).

28. J. R. O'Dea, N. J. Economou, and S. K. Buratto, Macromolecules, 46, 2267 (2013).

29. M. A. Modestino, A. Kusoglu, A. Hexemer, A. Z. Weber, and R. A. Segalman, Macromolecules, 45, 4681 (2012).

30. M. A. Modestino, D. K. Paul, S. Dishari, S. A. Petrina, F. I. Allen, M. A. Hickner, K. Karan, R. A. Segalman, and A. Z. Weber, Macromolecules, 46, 867 (2013).

31. E. Aleksandrova, R. Hiesgen, K. A. Friedrich, and E. Roduner, Phys. Chem. Chem Phys., 9, 2735 (2007)

32. E. Aleksandrova, R. Hiesgen, K. A. Friedrich, and E. Roduner, Phys. Chem. Chem. Phys., 9, 2735 (2007)

33. R. Hiesgen, S. Helmly, T. Morawietz, X.-Z. Yuan, H. Wang, and K.A. Friedrich, Electrochimica Acta, 110, 292 (2013)

34. B. V. Derjaguin, V. M. Muller, and Y. P. Toporov, J. Colloid Interface Sci., 53, 314 (1975).

35. A. Kusoglu, M. A. Modestino, A. Hexemer, R. A. Segalman, and A. Z. Weber, ACS Macro Lett., 1, 33 (2011).

36. S. Goswami, S. Klaus, and J. Benziger, Langmuir, 24, 8627 (2008)

37. M. Bass, A. Berman, A. Singh, O. Konovalov, and V. Freger, J. Phys. Chem. B, 114, $3784(2010)$

38. J. Tang, W. Yuan, J. Zhang, H. Li, and Y. Zhang, RSC Adv, 3, 8947 (2013).

39. M. H. Eikerling and P. Berg, Soft Matter, 7, 5976 (2011).

40. B. Loppinet and G. Gebel, Langmuir, 14, 1977 (1998). 